\title{
Review of pemetrexed in combination with cisplatin for the treatment of malignant pleural mesothelioma
}

\author{
Ranjit K Goudar \\ Department of Medicine, Division of \\ Hematology, Medical Oncology and \\ Cellular Therapy, Duke University \\ Medical Center, Durham, NC, USA
}

\begin{abstract}
Malignant pleural mesothelioma is a resistant form of lung cancer, and its incidence continues to rise in Europe and Australia. Until recently, chemotherapy had not been shown to be effective in the treatment of this slowly progressive disease. In 2004, the combination of pemetrexed and cisplatin was shown to induce high response rates in MPM. This article reviews the published literature describing the development and testing of this therapeutic combination in mesothelioma, and examines in detail the key phase III clinical trial that led to the approval of pemetrexed by the US FDA. Ongoing research will further define the role of pemetrexed plus cisplatin in the treatment of MPM.
\end{abstract}

Keywords: malignant pleural mesothelioma, mesothelioma, pemetrexed, cisplatin

\section{Methodology}

A systematic review of the literature was performed for published articles describing the development and testing of the combination of pemetrexed and cisplatin in the treatment of malignant pleural mesothelioma. Selected references and conference abstracts were also reviewed.

\section{Introduction}

\section{Epidemiology}

Malignant pleural mesothelioma (MPM) is a slowly progressive tumor involving the lining of the lungs; approximately $80 \%$ of cases are linked to prior asbestos exposure (Robinson et al 2005). In the US, approximately 2500 new cases of mesothelioma are diagnosed each year (Weill et al 2004). Asbestos use was banned in the US in 1971, and so the incidence of mesothelioma in the US is expected to continue to steadily decline.(Hazarika et al 2005) In contrast, asbestos use continued in Australia until 2003 and in Europe until 2005. As a result, the incidence of mesothelioma in European countries continues to rise and is projected to peak in 2020, and may account for as many as 250,000 European deaths in the next 35 years (Gatzemeier 2004). In 2020, over 18,000 Australian cases will be diagnosed (Hazarika et al 2005). Over the next 40 years, the direct and indirect costs of mesothelioma care will total over $\$ 200$ billion in the US alone, making MPM therapy an important public health issue (Robinson and Lake 2005).

\section{Prognosis}

MPM carries an extremely poor prognosis, and the median survival of patients with MPM is often as little as 7 months (Budde and Hanna 2004). Due to the insidious and progressive nature of this cancer, only $10 \%-15 \%$ of patients have resectable disease 
at presentation (Budde and Hanna 2004). In addition, the lengthy delay between asbestos exposure and the development of MPM mean that patients are often older and have significant comorbidities that render them poor surgical candidates at the time of diagnosis. As a result, chemotherapeutics have become the mainstay of disease therapy.

\section{Cisplatin and standard chemotherapy}

As recently as 2003, single-agent chemotherapy regimens produced response rates below 20\% in MPM (Pistolesi and Rusthoven 2004), and single agents had not been shown to improve median survival. Given the poor response of MPM to traditional chemotherapy regimens, the British Thoracic Society only recommended chemotherapy within the context of clinical trials as recently as 2001 (British Thoracic Society 2001).

Cisplatin has long been a mainstay in the treatment of lung cancer, including MPM. Zidar et al reported a 14.3\% response rate to single-agent cisplatin (Zidar et al 1988). In a subsequent meta-analysis, Berghmans et al (2002) reported response rates of $22.6 \%$ with combination chemotherapy, significantly higher than the $11.6 \%$ response from single-agent regimens $(p<0.001)$. Cisplatin was the most active single agent reviewed, with a response rate of $23 \%$. The combination of cisplatin and doxorubicin was the most active doublet, with a response rate of $28.5 \%$. Almost all of these trials enrolled fewer than 50 patients, calling the reproducibility of these results into question. While median survival is the most clinically relevant endpoint of chemotherapy testing, the bulk of pemetrexed trials prior to 2003 were phase I or II studies, forcing Berghmans et al to use response rate as the key endpoint in their meta-analysis (Berghmans et al 2002; Budde and Hanna 2004) .

\section{Pemetrexed}

\section{Mechanism of action}

Proliferating tumor cells depend on de novo nucleotide synthesis for their survival, and antimetabolite compounds such as pemetrexed are designed to inhibit nucleotide production (Curtin and Hughes 2001). This effect is reversible; preclinical testing showed that exogenous hypoxanthine and thymidine were necessary to prevent growth inhibition by pemetrexed (Curtin and Hughes 2001).

Lower levels of pemetrexed (Eli Lilly and Company, Indianapolis, IN, USA) inhibit thymidylate synthase and dihydrofolate reductase, while higher levels inhibit glycinamide ribonucleotide formyltransferase, inhibiting purine synthesis (Hazarika et al 2005). In human leukemia cells, treatment with pemetrexed resulted in lower concentrations of dTTP, dGTP and dCTP (Curtin and Hughes 2001).

Pemetrexed monoglutamate is transported into cells by the reduced folate carrier, and is subsequently polyglutamated by the folylpolygamma-glutamate synthetase (Hazarika et al 2005). The addition of multiple glutamate moieties enables the compound to be retained in the cell for longer periods, and increases the compound's affinity for cellular enzymes needed for the de novo synthesis of adenosine, guanine and thymidine (Hazarika et al 2005). For example, the pentaglutamatation of pemetrexed increases its ability to inhibit thymidylate synthase 84-fold (Curtin and Hughes 2001).

\section{Pharmacokinetics}

Pemetrexed is renally excreted, with a half-life of 3.5 hours (Hazarika et al 2005). After intravenous pemetrexed infusion, $90 \%$ of the original dose is excreted unmetabolized in the urine within 24 hours (Hazarika et al 2004). Pemetrexed has not been tested in patients with creatinine clearance under $45 \mathrm{~mL} /$ minute, and so should not be used in these patients (Hazarika et al 2005). Nonsteroidal anti-inflammatory drug (NSAID) use may decrease renal clearance of pemetrexed, and should be avoided around the time of pemetrexed administration (Hughes et al 2002).

\section{Pemetrexed plus cisplatin Preclinical studies}

In preclinical testing, the combination of pemetrexed and cisplatin showed activity against human non-small cell lung cancer cell lines, suggesting potential efficacy in MPM. When comparing cisplatin or pemetrexed alone versus combination treatment in $\mathrm{H} 460$ human non-small cell lung carcinoma (NSCLC) and Calu-6 NSCLC nude mouse xenografts, a greater-than-additive growth delay was noted (Teicher et al 2000). Pemetrexed was then studied in human patients with solid tumors.

\section{Phase I evaluation}

In phase I studies, patients with MPM, NSCLC, GI, and breast tumors responded to pemetrexed alone (Pearce and Alice Miller 2005). In a dose-escalation phase I study of patients with various solid tumors, 42 patients were treated with pemetrexed and cisplatin on day 1 of a 21-day cycle, while 12 patients were treated with pemetrexed on day 1 and cisplatin on day 2 of a 21-day cycle (Thodtmann et al 1999). 
More toxicity, including 2 deaths from neutropenic sepsis, were noted in the split-dosing regimen, so the authors recommended that pemetrexed and cisplatin be administered on the same day (Thodtmann et al 1999). Five of 13 patients with MPM in this study had a partial response to the combination of pemetrexed and cisplatin (Thodtmann et al 1999).

The maximum tolerated dose of pemetrexed $600 \mathrm{mg} / \mathrm{m}^{2}$ and cisplatin $100 \mathrm{mg} / \mathrm{m}^{2}$, was limited by neutropenia and leukopenia.(Eli Lilly and Company 2004a) Transaminitis and rash were also noted; pretreatment with glucocorticoids reduced the incidence of rash (Adjei et al 2000). Based on the results of this trial, pemetrexed $600 \mathrm{mg} / \mathrm{m}^{2}$ and a recommended dose of cisplatin $75 \mathrm{mg} / \mathrm{m}^{2}$ were recommended (Eli Lilly and Company, 2004a), and further study of this combination in MPM was planned.

\section{Phase II evaluation}

A phase II trial of pemetrexed and cisplatin was not conducted in patients with mesothelioma. Based on the Thodtmann phase I trial, Shepherd et al (2001) conducted a phase II trial of pemetrexed and cisplatin for patients with untreated stage IIIB (5/31) or IV (26/31) non-small cell lung cancer. Due to toxicity observed in phase I studies, the dose of pemetrexed was lowered to $500 \mathrm{mg} / \mathrm{m}^{2}$. A response rate of $45 \%$ (95\% CI 26\%-64\%) was noted, with a median duration of response of 6.1 months. The median survival was 8.9 months (Shepherd et al 2001). Overall, the combination of cisplatin and pemetrexed was well tolerated; patients received $98.0 \%$ of the planned dose intensity of cisplatin, and $96.0 \%$ of the planned dose intensity of pemetrexed (Shepherd et al 2001). Although one-third of the patients developed grade 3/4 granulocytopenia, only one case of neutropenic fever was recorded (Shepherd et al 2001). Based on these data, the same doses of cisplatin and pemetrexed were used in a subsequent phase III trial for MPM.

\section{Phase III evaluation EMPHACIS trial design}

After promising results from phase I and II pemetrexed trials, a patient-blinded phase III trial (Evaluation of Mesothelioma in a Phase III Trial of Pemetrexed with Cisplatin, EMPHACIS) randomized 448 chemonaive patients with MPM to pemetrexed $\left(500 \mathrm{mg} / \mathrm{m}^{2}\right)$ and cisplatin $\left(75 \mathrm{mg} / \mathrm{m}^{2}\right)$ every 21 days versus cisplatin alone (Vogelzang et al 2003). This was the largest mesothelioma trial to date, and these patients were followed for a median of ten months (Vogelzang et al 2003). The use of cisplatin alone as a control has since been criticized because cisplatin doublets were considered to be the standard of care prior to this trial (Steele 2003).

While EMPHACIS was underway, Niyikiza et al (2002) reported that elevated levels of homocysteine and methylmalonic acid, markers of folate and vitamin $\mathrm{B}_{12}$ deficiency, were associated with increased rates of side effects from pemetrexed. Patients with both homocysteine and methylmalonic acid in the highest quartile were 15.6 times more likely to suffer severe toxicity than those with normal levels. Due to this analysis, the decision was made mid-trial to administer folate and vitamin $\mathrm{B}_{12}$ supplementation to all subsequently enrolled patients in an effort to reduce rates of neutropenia and leukopenia. At that point, 70 patients had completed the trial without receiving vitamin supplementation. Forty-seven patients began supplementation partway through the trial, and 448 patients were subsequently enrolled and received vitamin supplements during their entire treatment course. Subsequent analysis of the data from this trial was separated by treatment arm and also by vitamin supplementation status; however, the relatively small number of never supplemented/partially supplemented complicates efforts to understand the effect of supplementation.

Vitamin supplementation did reduce the toxicity of pemetrexed; $52 \%$ of unsupplemented/partially supplemented patients reported grade 3 or 4 neutropenia, compared to only $9.4 \%$ of fully supplemented patients. Fully supplemented patients received a median of 6 cycles of pemetrexed, compared to 2 cycles of treatment for patients who did not receive any vitamin supplementation (Vogelzang et al 2003).

\section{EMPHACIS patient characteristics}

Due to limited treatment options and poor outcomes in MPM, significant pressure exists to expand the application of novel therapies to an ever-broader patient population, but overgeneralization increases the risk of poor outcomes. For this reason, it is important to examine the characteristics of patients included in the EMPHACIS trial. EMPHACIS enrolled patients who had unresectable disease or were not surgical candidates, who had not received prior chemotherapy, and with Karnofsky performance scores $\geq 70$. Most MPM patients are more symptomatic and less functional than this at presentation (Green et al 2007), which may limit the ability to apply this regimen to other patients.

Twelve percent of patients had received radiation therapy prior to enrollment.

Over $80 \%$ of patients were male and over $90 \%$ Caucasian (Vogelzang et al 2003). While $78 \%$ of the enrolled patients had advanced stage III or IV disease, $85 \%$ had epithelial 
histology (Vogelzang et al 2003), which has been associated with favorable outcome in EPP patient series (Sugarbaker et al 1999).

\section{EMPHACIS results}

The median survival of patients in the combination arm was 12.1 months, compared to a median survival of 9.3 months in cisplatin-only patients $(\mathrm{p}=0.02)$ (Vogelzang et al 2003). Intention-to-treat analysis was not upheld, since eight patient data points were absent (Steele and Klabatsa 2005). A subgroup analysis of patients who received vitamin supplementation throughout their chemotherapy course showed that the median survival of combination-arm patients was 13.3 months, compared to 10.0 months for cisplatin-only patients; however, the difference in median survival in this subgroup was no longer statistically significant $(\mathrm{p}=0.051)$. The hazard ratio for death for patients treated with both pemetrexed and cisplatin was 0.77 . In addition, the median time to progression was 5.7 months in the combination arm, and 3.9 months for cisplatin alone $(p=0.001)$. The total response rate was $41.3 \%$ in the combination arm, and $16.7 \%$ for cisplatin alone $(\mathrm{p}<0.0001)$. No complete responses were noted (Vogelzang et al 2003).

In 2005, the final data from EMPHACIS showed that the median survival of patients receiving pemetrexed and cisplatin was 12.8 months, compared to 9.0 months for those receiving cisplatin alone. Patients receiving combination therapy had a hazard ratio for death of 0.74 (95\% CI 0.60-0.90, p 0.003) (Vogelzang et al 2005). The US Food and Drug Administration (FDA) performed an intention-totreat analysis of the EMPHACIS data, and their results also favored combination therapy with cisplatin and pemetrexed (Hazarika et al 2004).

Patients who achieved an objective response in EMPHACIS had more improvement in pulmonary function tests (PFTs) than those with stable disease; in comparison, those who progressed on this regimen had a mean decrease in their $\mathrm{FEV}_{1}$ and FVC (Paoletti 2003). The differences in $\mathrm{FEV}_{1}$ and FVC between responders, those with stable disease, and those who progressed were all statistically significant. The authors commented that PFTs may be a more sensitive indicator of response to this disease, which is difficult to assess radiographically (Paoletti 2003).

\section{Cisplatin and EMPHACIS}

Outcomes in the cisplatin-only arm were unexpectedly poor compared to other trials. Although different enrolled populations make it difficult to compare survival rates between two clinical trials, concern exists that the low median survival of the EMPHACIS cisplatin-only arm may have made combination therapy appear more effective. The EORTC trial of cisplatin $80 \mathrm{mg} / \mathrm{m}^{2}$ with and without raltitrexed $3 \mathrm{mg} / \mathrm{m}^{2}$ did not find a statistically significant difference between these two arms. Reck and Gatzemeier (2005) attribute the difference in results of EMPHACIS and the EORTC trial to the fact that the cisplatin-only arm in EORTC showed a 39.4\% one-year survival, as opposed to the $17 \%$ one-year survival in the cisplatin-only arm in EMPHACIS. In addition, the EMPHACIS protocol did not specify a goal number of cycles, and the fact that control patients received a median of 2 less cycles than patients in the combination arm may account in part for the difference in survival between the two arms (Green et al 2007).

Despite the rosy $23 \%$ overall response rate of single-agent cisplatin in Berghmans' meta-analysis (Berghmans et al 2002). an early phase II trial showed that cisplatin alone led to a response rate of only $14.3 \%$ and median survival of 7.5 months in unresectable MPM (Zidar et al 1988). Based on these numbers, Budde and Hanna (2004) query the relative contribution from cisplatin to the activity of the cisplatin plus pemetrexed doublet, going so far as to propose a phase III trial of pemetrexed alone.

\section{Post-study chemotherapy after EMPHACIS}

The incidence of post-study chemotherapy may have affected the survival times of patients in the EMPHACIS study. $37.6 \%$ of patients who received cisplatin and pemetrexed and $47.3 \%$ of the cisplatin-only patients received a different post-study chemotherapy (Vogelzang et al 2003). Patients in the cisplatin-only arm were prohibited from receiving pemetrexed after the conclusion of the trial. Single-agent gemcitabine was the most commonly used post-study chemotherapy (PSC).

The median survival of patients treated with cisplatin plus pemetrexed and subsequent PSC was 15.3 months (95\% CI 13.3-18.9 months), compared to median survival of 12.2 months (95\% CI 9.9-14.2 months) in patients treated with cisplatin and pemetrexed without PSC. The median survival for combination-arm patients who did not receive PSC was 9.8 months (95\% CI 8.1-11.7 months), and 6.8 months (95\% CI 6.3-8.7 months) for cisplatin-only patients without PSC.

Receiving post-study chemotherapy was associated with increased survival ( $\mathrm{p}<0.001$ ) even after adjusting for patient prognostic factors and controlling for initial study regimen. Due to confounding factors, it is difficult to interpret 
this survival benefit, which may have been due to patients with less aggressive disease surviving to receive additional chemotherapy, due to either the initial or the subsequent chemotherapy regimens, or related to the elapsed interval between EMPHACIS regimen and receiving PSC (Manegold et al 2005).

\section{Toxicity}

When patients enrolled in the phase III EMPHACIS trial were supplemented with 350-1000 mg of oral folic acid daily and $1000 \mu \mathrm{g}$ of vitamin B12 via intramuscular injection every 9 weeks, their average homocysteine level fell from 8.3 to $6.9 \mu \mathrm{mol} / \mathrm{L}$. In addition, the rate of grade 4 hematologic or grade $3 / 4$ nonhematologic toxicity in these supplemented patients was $6.4 \%$, compared to $37 \%$ in unsupplemented patients (Bunn et al 2001). These results show that vitamin supplementation lowers homocysteine levels and reduces the incidence of toxicity from pemetrexed.

The original maximum tolerated dose of pemetrexed was calculated without benefit of vitamin supplementation. In a more recent phase I trial attempting to define the maximum tolerated dose of pemetrexed alone with vitamin B12 and folate supplementation, patients were treated with up to $800 \mathrm{mg} / \mathrm{m}^{2}$ of pemetrexed with acceptable toxicity (Nakagawa et al 2003). It is possible that if additional trials were performed with vitamin support, the maximum tolerated dose of cisplatin plus pemetrexed might well involve even higher doses of pemetrexed (Budde and Hanna 2004) and perhaps even higher response rates than those seen in EMPHACIS.

\section{FDA approval}

When independent reviewers and the FDA retrospectively assessed the radiographic response data from the phase III trial of pemetrexed and cisplatin versus cisplatin alone (Vogelzang et al 2003), the FDA only confirmed 47 of 94 partial responses reported by the study authors in the combination arm. A Cochrane review of pemetrexed and cisplatin for the treatment of mesothelioma raised concern that the lack of investigator blinding in EMPHACIS may have contributed to the discrepancy between trial investigators' assessment of response and that of independent FDA reviewers.

Since the FDA reviewers still noted more responses with combination therapy during their reassessment, pemetrexed was subsequently approved for the treatment of MPM in the United States on 4 February 2004 (Hazarika et al 2005). Due to the large number of patients and multiple international centers involved in EMPHACIS, the FDA approved pemetrexed without requiring a 2 nd supporting clinical trial demonstrating efficacy of cisplatin plus pemetrexed (Hazarika et al 2004).

Pemetrexed $500 \mathrm{mg} / \mathrm{m}^{2}$ was approved in combination with cisplatin $75 \mathrm{mg} / \mathrm{m}^{2}$ for the treatment of unresectable MPM (Hazarika et al 2005). The FDA also recommended that patients begin supplementation with folate 350-1000 $\mu \mathrm{g}$ daily and vitamin B12 $1000 \mathrm{mcg}$ intramuscular every 9 weeks 3 weeks prior to pemetrexed therapy. In order to reduce the incidence of pemetrexed-associated rash, dexamethasone pretreatment was recommended on days 0,1 , and 2 of chemotherapy (Hazarika et al 2005).

\section{Quality of life}

With the advent of targeted chemotherapy agents that may stabilize tumor growth as opposed to causing clinical regression, other outcomes measures such as quality of life will be more important than ever. In a phase II trial of cisplatin and pemetrexed in MPM, 32\% of patients showed improvement in their Lung Cancer Symptom Scale score (Eli Lilly and Company 2004b).

The LCSS-Meso questionnaire has been previously validated in mesothelioma patients (Hollen et al 2004). LCSSMeso was administered to patients in the EMPHACIS trial, with a $90 \%$ completion rate (Gralla et al 2003). The overall symptom score favored the combination arm after 6 cycles ( $\mathrm{p}=0.004)$ (Boyer et al 2003), and patients in that arm received a median of 6 cycles (Vogelzang et al 2003).

By week 12 (4 cycles), a statistically significant improvement in pain, cough and dyspnea was noted in the pemetrexed plus cisplatin arm, compared with the cisplatin-only arm. By week 12, similar improvements global quality of life $(p=0.025)$ and fatigue $(p=0.027)$ favoring the combination arm were noted. Anorexia scores favored the combination arm by week $18(\mathrm{p}=0.017)$ (Gralla et al 2003).

\section{Ongoing/future trials}

Based on the results of EMPHACIS, cisplatin and pemetrexed should be the standard of care (Ellis et al 2006) and thus the default comparator for testing the activity of future chemotherapy regimens against MPM (Reck and Gatzemeier 2005). Additional clinical trials are needed to better define the role of cisplatin and pemetrexed in the treatment of MPM, including the role of this doublet in combination with radiation therapy or surgical therapy such as pleurectomy/decortication and extrapleural pneumonectomy.

The Extended Access Program (EAP) study will provide nonrandomized data about pemetrexed, pemetrexed plus 
cisplatin, and pemetrexed plus carboplatin in patients with MPM. H3-MCJMEW compares best supportive care with or without pemetrexed. SITMP3 treats nonoperative patients with cisplatin, and those who do not progress are randomized to receive maintenance pemetrexed or pemetrexed at time of progression (Favaretto 2005).

\section{Pemetrexed plus carboplatin}

Carboplatin is often substituted for cisplatin due to its reduced risk of toxicity (Hughes et al 2002). In a phase I dose-escalation trial of 27 untreated patients with MPM, no dose-limiting toxicities (DLT) were noted in 3 patients treated with a combination of pemetrexed $500 \mathrm{mg} / \mathrm{m}^{2}$ and carboplatin area-under-the-curve (AUC) $5 \mathrm{mg} / \mathrm{mL} /$ minute every 21 days. All three of these patients developed at least grade 3 leukopenia. Three of 5 patients experienced DLT at pemetrexed $500 \mathrm{mg} / \mathrm{m}^{2}$ and carboplatin AUC 6 (2 patients with grade 4 neutropenia and 1 with neutropenic sepsis) (Hughes et al 2002). In this phase I trial, 8 of 25 assessable patients had a partial response (response rate 32\%), and 14 had stable disease (Hughes et al 2002). This response rate was higher than prior studies that reported response rates between 5 and 16 with single-agent carboplatin (Hughes et al 2002).

This phase I trial of carboplatin also reported a median survival of 451 days, with a median of 305 days to progression (Hughes et al 2002). The authors report that $70 \%$ of these patients described a decrease in "cancer-related symptoms" during chemotherapy, but the details of this measurement are not described (Hughes et al 2002). The mean GFR of these patients was $122 \mathrm{~mL} / \mathrm{min}$ prior to therapy and $93 \mathrm{~mL} /$ minute post-treatment $(\mathrm{p}<0.001)$, but the authors felt this decrease was not clinically significant (Hughes et al 2002).

A subsequent Italian phase II trial treated 102 patients with MPM with pemetrexed $500 \mathrm{mg} / \mathrm{m}^{2}$ and carboplatin area-under-the-curve (AUC) $5 \mathrm{mg} / \mathrm{mL} /$ minute every 21 days for a median of 6 cycles. All patients received vitamin B12 and folate supplementation. Two patients had complete responses, and 17 had a partial response, for a total response rate of $18.6 \%$ (95\% CI 11.6\%-27.5\%) (Ceresoli et al 2006), which was markedly lower than the $32 \%$ noted in the Hughes phase I study.

In the Ceresoli trial, 47\% (95\% CI 37.1\%-57.2\%) had stable disease. The median survival was 12.7 months, with median time to progression of 6.5 months. $19.6 \%$ of patients developed Grade 3-4 neutropenia, and 11.7\% experienced grade 3-4 anemia (Ceresoli et al 2006). Overall, the treatment was very well tolerated; of the 96 patients receiving over 2 cycles of pemetrexed and carboplatin, relative dose intensity was $97 \%$ for pemetrexed and $98 \%$ for carboplatin (Ceresoli et al 2006).

To date, there have been no published phase III data examining the efficacy of pemetrexed and carboplatin in the treatment of MPM.

\section{Conclusion}

The worldwide incidence of malignant pleural mesothelioma continues to rise, but treatment options have historically been limited. Surgical resection is technically difficult and quite morbid, and standard doublet chemotherapy has not been particularly effective. In recent years, the promising combination of pemetrexed plus cisplatin has been developed and tested in the treatment of MPM. The large phase III EMPHACIS trial showed improved outcomes in patients treated with both cisplatin and pemetrexed versus cisplatin alone. Ongoing and future testing will likely expand the role of this regimen in this disease. Combining cisplatin plus pemetrexed and other treatment modalities such as radiotherapy, surgical resection or targeted molecular therapies may lead to improved patient outcomes.

\section{References}

Adjei AA, Calvert AH, Hanauske AR, et al. 2000. Pemetrexed (ALIMTA), a novel multitargeted antifolate agent, is active alone and in combination in thoracic tumors. Lung Cancer, 29:38-9.

Berghmans T, Paesmans M, Lalami Y, et al. 2002. Activity of chemotherapy and immunotherapy on malignant mesothelioma:a systematic review of the literature with meta-analysis. Lung Cancer, 38:111-21.

Boyer M, Jassem J, Al, ALE. 2003. Symptoms and quality of life advantages for pemetrexed + cisplatin versus cisplatin in treatment of malignant pleural mesothelioma. Lung Cancer, 41:S19.

British Thoracic Society. 2001. Statement on malignant mesothelioma in the United Kingdom. Thorax, 56:250-65.

Budde LS, Hanna NH. 2004. Pemetrexed (Alimta.:improving outcomes in malignant pleural mesothelioma. Expert Rev Anticancer Ther, 4:361-8.

Bunn C, Paoletti P, Niyikiza C, et al. 2001. Vitamin B12 and folate reduce toxicity of Alimta ${ }^{\mathrm{TM}}$ (pemetrexed disodium, LY231514, MTA., a novel antifolate/antimetabolite. Proc Am Soc Clin Oncol, 20:abstr 300.

Ceresoli GL, Zucali PA, Favaretto AG, et al. 2006. Phase II study of pemetrexed plus carboplatin in malignant pleural mesothelioma. $J$ Clin Oncol, 24:1443-8.

Curtin NJ, Hughes AN. 2001. Pemetrexed disodium, a novel antifolate with multiple targets. Lancet Oncol, 2:298-306.

Eli lilly and Company. 2004a. Clinical Study Summary:Study H3EMC-JMAP.

Eli Lilly and Company. 2004b. Clinical Study Summary:Study H3EMC-JMDR.

Ellis P, Davies AM, Evans WK, et al. 2006. The use of chemotherapy in patients with advanced malignant pleural mesothelioma:a systematic review and practice guideline. J Thorac Oncol, 1:591-601.

Favaretto A. 2005. Overview on ongoing or planned clinical trials in Europe. Lung Cancer, 49(Suppl 1):S117-21.

Gatzemeier U. 2004. Pemetrexed in malignant pleural mesothelioma. Oncology (Williston Park), 18:26-31. 
Gralla RJ, Hollen PJ, Liepa AM, et al. 2003. Improving quality of life in patients with malignant pleural mesothelioma:Results of the randomized pemetrexed + cisplatin vs cisplatin trial using the LCSS-meso instrument. ASCO.

Green J, Dundar Y, Dodd S, et al. 2007. Pemetrexed disodium in combination with cisplatin versus other cytotoxic agents or supportive care for the treatment of malignant pleural mesothelioma. Cochrane Database Syst Rev:CD005574.

Hazarika M, White RM, Johnson JR, et al. 2004. FDA drug approval summaries:pemetrexed (Alimta). Oncologist, 9:482-8.

Hazarika M, White RM Jr, Booth BP, et al. 2005. Pemetrexed in malignant pleural mesothelioma. Clin Cancer Res, 11:982-92.

Hollen PJ, Gralla RJ, Liepa AM, et al. 2004. Adapting the Lung Cancer Symptom Scale (LCSS) to mesothelioma:using the LCSS-Meso conceptual model for validation. Cancer, 101:587-95.

Hughes A, Calvert P, Azzabi A, et al. 2002. Phase I clinical and pharmacokinetic study of pemetrexed and carboplatin in patients with malignant pleural mesothelioma. J Clin Oncol, 20:3533-44.

Manegold C, Symanowski J, Gatzemeier U, et al. 2005. Second-line (poststudy. chemotherapy received by patients treated in the phase III trial of pemetrexed plus cisplatin versus cisplatin alone in malignant pleural mesothelioma. Ann Oncol, 16:923-7.

Nakagawa K, Kudoh S, Matsui K, et al. 2003. A phase I dose-finding study of pemetrexed supplemented with folic acid (FA. and vitamin B12 in Japanese patients with solid tumors Proc Am Soc Clin Oncol, 22:22.

Niyikiza C, Baker SD, Seitz DE, et al. 2002. Homocysteine and methylmalonic acid:markers to predict and avoid toxicity from pemetrexed therapy. Mol Cancer Ther, 1:545-52.

Paoletti P, Pistolesi M, Rusthoven J, et al. 2003. Correlation of pulmonary function tests with best tumor response status:results from the phase III study of pemetrexed + cisplatin vs cisplatin in malignant pleural mesothelioma. Proc Am Soc Clin Oncol, 22:659.

Pearce HL, Alice Miller M. 2005. The evolution of cancer research and drug discovery at Lilly Research Laboratories. Adv Enzyme Regul, 45:229-55.

Pistolesi M, Rusthoven J. 2004. Malignant pleural mesothelioma:update, current management, and newer therapeutic strategies. Chest, 126:1318-29.
Reck M, Gatzemeier U. 2005. Pemetrexed-cisplatin combination in mesothelioma. Expert Rev Anticancer Ther, 5:231-7.

Robinson BW, Lake RA. 2005. Advances in malignant mesothelioma. N Engl J Med, 353:1591-603.

Robinson BW, Musk AW, Lake RA. 2005. Malignant mesothelioma. Lancet, 366:397-408.

Shepherd FA, Dancey J, Arnold A, et al. 2001. Phase II study of pemetrexed disodium, a multitargeted antifolate, and cisplatin as first-line therapy in patients with advanced nonsmall cell lung carcinoma:a study of the National Cancer Institute of Canada Clinical Trials Group. Cancer, 92:595-600.

Steele JP. 2003. The new front line treatment for malignant pleural mesothelioma? Thorax, 58:96-7.

Steele JP, Klabatsa A. 2005. Chemotherapy options and new advances in malignant pleural mesothelioma. Ann Oncol, 16:345-51.

Sugarbaker DJ, Flores RM, Jaklitsch MT, et al. 1999. Resection margins, extrapleural nodal status, and cell type determine postoperative longterm survival in trimodality therapy of malignant pleural mesothelioma:results in 183 patients. $J$ Thorac Cardiovasc Surg, 117:54-63; discussion $63-5$.

Teicher BA, Chen V, Shih C, et al. 2000. Treatment regimens including the multitargeted antifolate LY231514 in human tumor xenografts. Clin Cancer Res, 6:1016-23.

Thodtmann R, Depenbrock H, Dumez H, et al. 1999. Clinical and pharmacokinetic phase I study of multitargeted antifolate (LY231514. in combination with cisplatin. J Clin Oncol, 17:3009-16.

Vogelzang N, Symanowski J, Rusthoven J, et al. 2005. Long-term survival update from the randomized phase III study of pemetrexed plus cisplatin vs. cisplatin in patients with malignant pleural mesothelioma (MPM). Lung Cancer, 49:S230.

Vogelzang NJ, Rusthoven JJ, Symanowski J, et al. 2003. Phase III study of pemetrexed in combination with cisplatin versus cisplatin alone in patients with malignant pleural mesothelioma. J Clin Oncol, 21:2636-44

Weill H, Hughes JM, Churg AM. 2004. Changing trends in US mesothelioma incidence. Occup Environ Med, 61:438-41.

Zidar BL, Green S, Pierce HI, et al. 1988. A phase II evaluation of cisplatin in unresectable diffuse malignant mesothelioma: a Southwest Oncology Group Study. Invest New Drugs, 6:223-6. 
